\title{
Aspectos biológicos e pesqueiros de Paralonchurus brasiliensis Steindachner, (Pisces, Sciaenidae), na Armação do Itapocoroy, Penha, Santa Catarina, Brasil
}

\author{
Joaquim O. Branco', Maria J. Lunardon-Branco', José R. Verani ${ }^{2}$ \\ ${ }^{1}$ Centro de Ensino em Ciências Tecnológicas da Terra e do Mar, Universidade do Vale do Itajaí. Caixa Postal 360, \\ 88301-970 Itajaí, Santa Catarina, Brasil. E-mail: branco@univali.br \\ 2 Departamento de de Ecologia e Biologia Evolutiva, Universidade Federal de São Carlos. Caixa Postal 676, 13565-905 São \\ Carlos, São Paulo, Brasil.
}

\begin{abstract}
Biological and fishing aspects of Paralonchurus brasiliensis Steindachner, (Pisces, Sciaenidae), in the Armação do Itapocoroy, Penha, Santa Catarina, Brazil. Due to abundance of P. brasiliensis (Steindachner, 1875) in ictiofauna of the sea-bob-shrimp's bycatch, it presents low commercial value and frequently being discarded. This work aims to present basic information on biology and fisheries of P. brasiliensis in the Armação do Itapocoroy, Penha, Santa Catarina. The collections were carried out monthly during the period of August 1996 to July 2003, in three traditional areas of performance of artisan fisheries. The species presented positive alometric growth, with a sexual ratio of 1:2,1 between males and females, and the size of first maturation in 14,8 $\mathrm{cm}$ and $15,0 \mathrm{~cm}$. P. brasiliensis presented a great trophy specter composed by 27 items, being that, from the spring a gradual increment in the food ingestion occurred until the summer, followed of abrupt fall in the autumn and small recovery in the winter. Despite of the seasonal fluctuations during the seven years, this species occupied the second position in number and biomass among the integrant of ictiofauna bycatch.
\end{abstract}

WORDS KEY. Bycatch, ictiofauna, natural diet, population structure.

RESUMO. Apesar da abundância de Paralonchurus brasiliensis (Steindachner, 1875) na ictiofauna acompanhante do camarão sete-barbas, apresenta baixo valor comercial sendo freqüentemente descartada. Esse trabalho tem como objetivo apresentar informações básicas sobre a biologia e pesca de P. brasiliensis na Armação do Itapocoroy, Penha, Santa Catarina. As coletas foram realizadas mensalmente durante o período de agosto 1996 a julho 2003, em três áreas tradicionais de atuação da pesca artesanal. A espécie apresentou crescimento alométrico positivo, com uma razão sexual de 1:2,1 entre machos e fêmeas, e tamanho de primeira maturação em $14,8 \mathrm{~cm}$ e $15,0 \mathrm{~cm}$. P. brasiliensis apresentou amplo espectro trófico composto por 27 itens, sendo que, a partir da primavera ocorreu um incremento gradual na ingestão de alimento até o verão, seguido de queda abrupta no outono e de pequena recuperação no inverno. Apesar das flutuações sazonais ao longo dos sete anos, essa espécie ocupou a segunda posição em número e biomassa entre as integrantes da ictiofauna acompanhante.

PALAVRAS CHAVE. Dieta natural, estrutura populacional, fauna acompanhante, ictiofauna.

Paralonchurus brasiliensis (Steindachner, 1875), é um Sciaenidae, popularmente conhecido como Maria-Luiza, encontrado em profundidades inferiores a 100 metros sobre fundos de constituição arenosa, lamosa ou areno-lodosa, desde o Panamá à Argentina, Uruguai Venezuela; no Brasil desde o delta do Amazonas até o Rio Grande do Sul (Menezes \& Figueiredo 1980). Habitats semelhantes aos encontrados nos locais tradicionais de pesca artesanal do camarão sete-barbas (BRANCO et al. 1999).

Estudos específicos sobre os aspectos biológicos de $P$. brasiliensis estão restritos, na sua maioria, ao litoral de São Paulo (Paiva-Filho \& Rossi 1980, Braga et al. 1985, Cunningham \& Diniz-Filho 1995) e a sobreposição de populações na costa Su-
deste-Sul (Paiva-Filho \& ZANi-TeIXeIra 1980). Devido à sua abundância na ictiofauna acompanhante do camarão sete-barbas, essa espécie não apresenta valor comercial significativo (PAIVAFilho \& Schimigelow 1986), mas é importante o conhecimento da sua biologia e encadeamento trófico nas áreas de pesca pela sua participação no equilíbrio dos ecossistemas em explotação (Coelho et al. 1986).

O presente trabalho tem como objetivo apresentar informações básicas sobre a biologia e pesca de $P$. brasiliensis, oriunda da ictiofauna acompanhante da pesca artesanal do camarão sete-barbas realizada na Armação do Itapocoroy, Penha, Santa Catarina.

Revista Brasileira de Zoologia 22 (4): 1063-1071, dezembro 2005 


\section{MATERIAL E MÉTODOS}

As coletas foram realizadas mensalmente durante o período de agosto 1996 a julho 2003, na Armação do Itapocoroy, Penha $\left(26^{\circ} 40^{\prime}-26^{\circ} 47^{\prime} \mathrm{S}\right.$ e $\left.48^{\circ} 36^{\prime}-48^{\circ} 38^{\prime} \mathrm{W}\right)$, em três áreas tradicionais de atuação da pesca artesanal do camarão sete-barbas. Foram utilizadas duas redes-de-arrastos com portas (doublé rigged), tracionadas por baleeira de 10,5 metros de comprimento com motor de $40 \mathrm{Hp}$, velocidade média de dois nós e duração média de uma hora por arrasto (Branco et al. 2002). Os exemplares foram etiquetados, acondicionados em sacos plásticos e mantidos em caixas térmicas com gelo. Após a coleta, os exemplares que não puderam ser processados foram mantidos em "freezer" até serem trabalhados.

No período entre agosto/1996 a julho/2003 foram registradas a média mensal da CPUE $(\mathrm{kg} / \mathrm{h}$, número de exemplares/h), obtidas com as informações das três áreas de coleta. A ANOVA (SoKAL \& Rohlf 1969) foi aplicada aos dados transformados pelo logaritmo natural das CPUE e do número de exemplares, por ano e sazonal, sendo testados quanto à homogeneidade da variância (teste de Bartelett) e de normalidade da distribuição (prova de Kolmorov-Smirnov). Na existência de diferenças significativas, o contraste das médias (teste Tuckey-Kramer) foi aplicado para indicar quais médias foram significativamente diferentes. Também, utilizou-se a ANOVA para testar a igualdade das médias dos itens alimentares oriundas do método dos pontos por estação do ano.

Entre agosto/1997 a julho/1998, foi aleatoriamente retirada, a cada mês, uma subamostra representativa $(\mathrm{n}=65)$ de $P$. brasiliensis. Desses exemplares, foram registrados o sexo, estádio de maturação, comprimento total (Lt) em $\mathrm{cm}$ e o peso total (Wt) em gramas e analisado o conteúdo estomacal.

O teste do $\chi^{2}$, ao nível de significância de $5 \%$ e n-1 graus de liberdade $(n=2)$, foi aplicado para verificar a possível diferença entre a proporção sexual, mensalmente e por classes de comprimento total da submostra, bem como para verificar a possível ocorrência de diferenças significativas entre o volume relativo de alimento consumido por machos e fêmeas (VAzzoler 1996).

A relação peso total/comprimento total foi estimada para machos e fêmeas (SAntos 1978). O teste " $\mathrm{t}$ " de Student ( $\mathrm{p}<$ $0,05)$ foi utilizado para verificar a existência de diferenças significativas entre os valores de " $b$ " para machos e fêmeas da relação peso/comprimento, após obtenção das respectivas variâncias das retas. O tamanho de primeira maturação gonadal $\left(\mathrm{Lt}_{\mathrm{PM}}\right)$ dos exemplares adultos, foi estimado graficamente para machos e fêmeas (VAzzoler 1981).

A identificação dos itens alimentares de $P$. brasiliensis foi realizada com auxílio de bibliografia especializada, aquelas presas que não puderam ser determinadas, devido ao elevado grau de digestão, foram consideradas como matéria orgânica não determinada.

O índice de repleção dos estômagos foi estimado de acordo com SANTOS (1978). Na análise quali-quantitativa foram aplicados os métodos dos Pontos $(\mathrm{MP})=($ volume relativo $)$ e da
Freqüência de Ocorrência (FO) (HyNEs 1950, HysLop 1980, BERG 1979), complementados pelo índice alimentar (IAi) modificado de KaWAKami \& VAzzoler (1980), o que contribuiu para a compreensão global da dieta, tornando possíveis comparações com outras espécies e as inter-relações predador-presa. A diversidade e a equitabilidade foram estimados e aplicados aos itens alimentares consumidos pela espécie (LuDWig \& REYNolds 1988).

\section{RESULTADOS}

Na distribuição da freqüência absoluta dos exemplares subamostrados, as fêmeas dominaram significativamente $\left(\chi^{2}\right.$, $\mathrm{p}<0,05)$ em 11 meses; apenas em julho/98 ocorreu um equilíbrio entre os sexos (Fig. 1). A razão sexual, ao longo do ano foi de 1:2,1 a favor das fêmeas. A distribuição da freqüência relativa por classe de comprimento total (Fig. 2), apresentou uma tendência relativamente semelhante entre os sexos, com diferença significativa a favor dos machos na classe de $18,0 \mathrm{~cm}$ e das fêmeas em $23,0 \mathrm{~cm}$.

Nos 787 exemplares subamostrados, em 1997-1998 com uma média mensal de 65,6 peixes, a amplitude de comprimento total variou entre 2,0 a $28,0 \mathrm{~cm}$, com comprimento médio de $15,9 \pm 4,4 \mathrm{~cm}$, onde aproximadamente $34,4 \%$ dos $P$. brasiliensis apresentaram comprimento inferior a $18,0 \mathrm{~cm}$ (Fig. 3).

A determinação macroscópica do sexo foi possível a partir de 11,0 cm de comprimento total, sendo que as fêmeas atingiram tamanho superior ao dos machos, com as maiores freqüências ocorrendo, respectivamente, nas classes de 20,0 a 22,0 e 18,0 a 20,0 cm, enquanto que nos exemplares de sexo indeterminado ficou entre 10,0 a 13,0 cm (Fig. 3). Entre os comprimentos de 11,0 a $18,0 \mathrm{~cm}$, a separação dos sexos através da inspeção macroscópica das gônadas não foi muito eficiente. $P$. brasiliensis são peixes pertencentes a populações de pequeno porte, com comprimento médio em torno de $16,0 \pm 4,4 \mathrm{~cm}$.

O tamanho de primeira maturação gonadal foi estimado entre $14,8 \mathrm{~cm}$ (machos) e 15,0 cm (fêmeas) de comprimento total; a partir dos $18,0 \mathrm{~cm}$, todos os peixes analisados eram adultos (Fig. 3).

A relação peso/comprimento total dos exemplares subamostrados indicou um padrão de crescimento relativo alométrico positivo nos machos ( $\mathrm{Wt}=0,0031 \mathrm{Lt}^{3,3676}, \mathrm{r}^{2}=0,9048$ ) e nas fêmeas (Wt $\left.=0,0029 \mathrm{Lt}^{3,3970}, \mathrm{r}^{2}=0,9531\right)$, isométrico nos exemplares de sexo indeterminado ( $\mathrm{Wt}=0,0063 \mathrm{Lt}^{3,0617}, \mathrm{r}^{2}=0,9484$ ); não ocorrendo diferença significativa entre os valores de " $\mathrm{b}$ " machos-fêmeas ( $\mathrm{t}=0,0043 ; \mathrm{p}<0,05, \mathrm{gl}=514$ ). Independente do sexo e estágio de maturação, parece que a população tende ao padrão alométrico positivo ( $\mathrm{Wt}=0,0033 \mathrm{Lt}^{3,3468}, \mathrm{r}^{2}=0,9805$ ).

Foram analisados 787 estômagos de $P$. brasiliensis; destes, 163 retirados dos machos, 353 das fêmeas e 271 dos exemplares de sexo indeterminado, sendo que 76,08\%, 79,04 e 58,67\%, respectivamente, continham algum tipo de alimento (Tab. I).

O teste do $\chi^{2}$ aplicado sobre a freqüência percentual de pontos nas 10 categorias alimentares, incluindo matéria orgânica e areia, não apresentou diferença significativa entre os itens

Revista Brasileira de Zoologia 22 (4): 1063-1071, dezembro 2005 

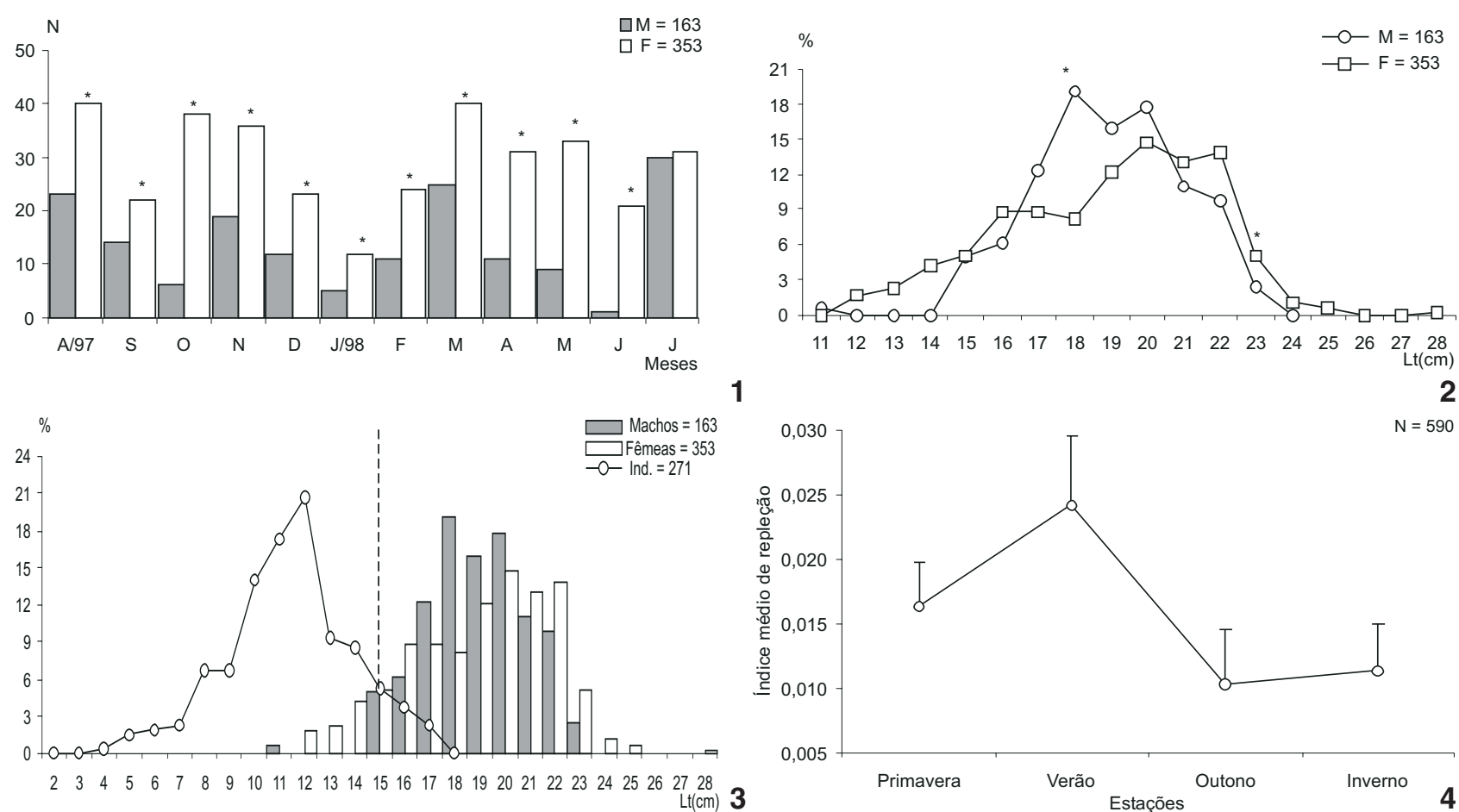

Figuras 1-4. Paralonchurus brasiliensis em 1997-1998. 1-2. Distribuição mensal da (1) freqüência de ocorrência e (2) percentual por classe de comprimento total (\%) de machos e fêmeas. (*) Diferença significativa, $\chi^{2}(p<0,05)$; (3) freqüência percentual por classe de comprimento para fêmeas, machos e indeterminados . A linha tracejada indica o tamanho de primeira maturação: fêmeas - $\mathrm{Lt}_{\mathrm{PM}}=14,8 \mathrm{~cm}$; machos - $\mathrm{Lt}_{\mathrm{PM}}$ = 15,0 cm; (4) flutuação sazonal do índice médio de repleção dos estômagos. As barras verticais correspondem ao erro da média.

Tabela I. Distribuição de freqüência de ocorrência de $P$. brasiliensis machos, fêmeas e jovens de sexo indeterminados com estômagos vazio e com conteúdo, durante o período de julho/1997 a junho/1998.

\begin{tabular}{lccccccc}
\hline \multirow{2}{*}{ Sexo } & \multicolumn{2}{c}{$\begin{array}{c}\text { Estômagos } \\
\text { vazios }\end{array}$} & & \multicolumn{3}{c}{$\begin{array}{c}\text { Estômagos com } \\
\text { conteúdo }\end{array}$} & \multirow{2}{*}{ Total } \\
\cline { 2 - 3 } & $\mathrm{N}$ & $\%$ & & $\mathrm{~N}$ & $\%$ & \\
\hline Machos & 39 & 23,92 & & 124 & 76,08 & 163 \\
Fêmeas & 74 & 20,96 & & 279 & 79,04 & 353 \\
Indeterminados & 112 & 41,33 & & 159 & 58,67 & 271 \\
\hline
\end{tabular}

consumidos por machos e fêmeas (Tab. II), assim a dieta natural da espécie foi analisada para sexos agrupados, acrescido dos exemplares de sexo indeterminado.

O índice médio de repleção dos estômagos, revelou a ocorrência de flutuações sazonais na dieta da espécie (Fig. 4). A partir da primavera ocorreu um incremento gradual na ingestão de alimento até o verão, seguido de queda abrupta no outono e de pequena recuperação no inverno.

Através da análise do conteúdo estomacal dos exemplares, foi possível identificar 27 itens componentes da dieta natural da espécie (Tab. III). Na primavera, os itens mais explora- dos foram Flabelligeridae, Dendrobranchiata, Acetes americanus Ortmann, 1893 e Polychaeta. Nessa estação, ocorreu a maior diversidade no ano $(1,9806)$, com uma equitabilidade $(69,90 \%)$, indicando que os itens alimentares foram explorados com elevada uniformidade.

No verão, embora $P$. brasiliensis tenha utilizado 16 itens na sua dieta, como na primavera, ocorreram pequenas alterações na composição, onde os Osteichthyes e Pleoticus müllieri (Bate, 1888) foram o segundo e quarto recursos, respectivamente, mais utilizados (Tab. III). A diversidade foi a menor do ano com uma equitabilidade em torno de $61,95 \%$, demonstrando que os itens não foram explorados uniformemente, principalmente pelas baixas ocorrências dos Nematoda, Copepoda, areia e Gamaridae.

No outono, apesar da redução do número de presas consumidas e da dominância dos Flabelligeridae, A. americanus, matéria orgânica e Dendrobranchiata; com uma diversidade discretamente superior ao verão, ocorreu a maior equitabilidade (73,54\%) do ano (Tab. III), apresentando maior uniformidade na utilização dos itens alimentares quando comparado às demais estações.

No inverno, a espécie ampliou seu espectro trófico para 23 itens, onde os caranguejos Pinnotheridae ocuparam a segunda posição, além de ter acrescentado recursos novos na di- 
Tabela II. Freqüência absoluta dos pontos (MP) e percentual (\%) das categorias alimentares na dieta de machos e fêmeas de $P$. brasiliensis. Teste do $\chi^{2}$ entre as freqüências percentuais dos pontos.

\begin{tabular}{|c|c|c|c|c|c|}
\hline \multirow{2}{*}{ Categorias Alimentares } & \multicolumn{2}{|c|}{ Machos } & \multicolumn{2}{|c|}{ Fêmeas } & \multirow{2}{*}{$\chi^{2}$} \\
\hline & MP & $\%$ & MP & $\%$ & \\
\hline Algae & - & - & 2 & 0,01 & - \\
\hline Cnidaria & - & - & 25 & 0,12 & - \\
\hline Nematoda & 5 & 0,05 & 2 & 0,01 & 0,030 \\
\hline Mollusca & - & - & 17 & 0,08 & - \\
\hline Polychaeta & 5544 & 55,82 & 13682 & 64,75 & 0,660 \\
\hline Crustacea & 3582 & 36,07 & 5752 & 27,22 & 1,240 \\
\hline Echinodermata & 250 & 2,52 & 509 & 2,41 & 0,002 \\
\hline Osteichthyes & 150 & 1,51 & - & - & - \\
\hline Matéria Orgânica & 399 & 4,02 & 1137 & 5,38 & 0,200 \\
\hline Areia & 2 & 0,02 & 6 & 0,03 & 0,001 \\
\hline Total & 9932 & 100,00 & 21132 & 100,00 & \\
\hline
\end{tabular}

eta como Algae, Octocorallia, Pitar fulminatus (Menke, 1828), ovos de Crustácea, entre outros (Tab. III); apesar desse incremento, a diversidade manteve-se em $(1,9356)$, com a menor equitabilidade do ano (60,90\%), demonstrando que não houve uma uniformidade na exploração dos recursos.

Embora tenham ocorrido alterações sazonais entre o número e intensidade dos itens presas exploradas, não foram observadas diferenças significativas $\left(\mathrm{F}_{3-66}=0,2861 ; \mathrm{p}<0,05\right)$ entre o volume médio de alimento consumido e as estações do ano.

A figura 5, apresenta a contribuição em freqüência de ocorrência, pontos e o índice alimentar, durante o período de agosto/97 a julho/98. Crustacea foi a categoria mas freqüente na dieta de $P$. brasiliensis, enquanto que, os Polychaeta dominaram em pontos. De acordo com o IAi, os Polychaeta $(0,5528)$ e Crustacea $(0,4219)$ constituíram o primeiro grupo trófico, Matéria Orgânica e Echinodermata o segundo, Osteichthyes o terceiro, Outros (Algae, Cnidaria, Nematoda, Mollusca e Areia), podem ser considerados de ocorrência acidental na dieta da espécie (Fig. 5, Tab. III).

Das 59 espécies integrantes da ictiofauna acompanhante da pesca artesanal do camarão sete-barbas na Armação do Itapocoroy, $P$. brasiliensis ocupou a segunda posição em número e biomassa. Sua participação relativa em biomassa esteve acima de $30,0 \%$ ao longo dos sete anos de coleta, com as maiores capturas ocorrendo em 96/97 e as menores em 02/03 (Fig. 6). Enquanto que a contribuição em número de exemplares representou entre 10,45 a 36,31\% da ictiofauna acompanhante (Fig. 7).

Os sete anos de coleta, quando reunidos em uma série temporal (Figs 8 e 9), indicam que ocorreram flutuações sazonais nas capturas de $P$. brasiliensis ao longo dos anos, com os maiores valores médios de CPUE (kg e número) ocorrendo entre os meses de setembro a janeiro e os menores entre abril a julho, exceto entre 2000-2001 e 2001-2002, que apresentaram capturas expressivas nos meses de outono-inverno. Apesar das

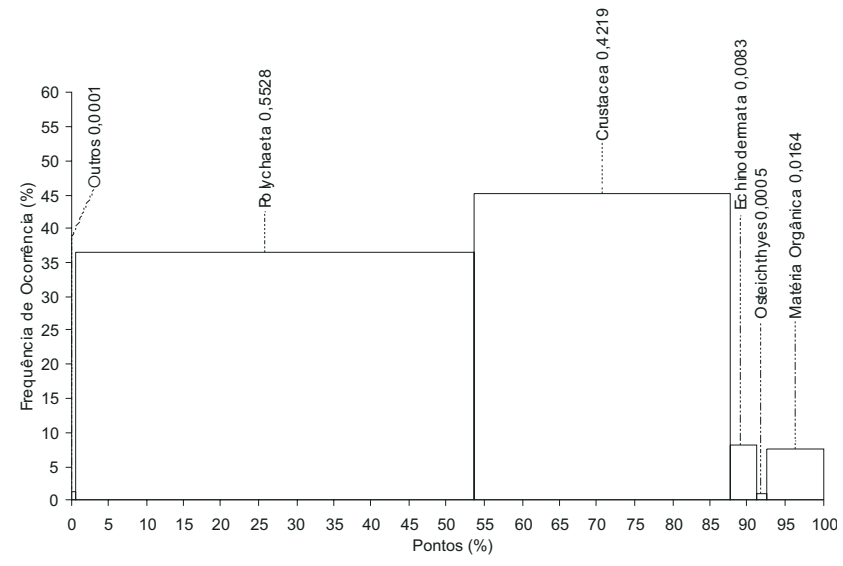

Figura 5. Freqüência de ocorrência (\%) e dos pontos (\%) das categorias alimentares e seus respectivos índices de $P$. brasiliensis, durante o período de estudo.(Outros = Algae, Cnidaria, Nematoda, Mollusca e Areia).

oscilações observadas nas séries temporais, não ocorreram diferenças significativas entre as CPUE médias dos sete anos de amostragens (kg: $\mathrm{F}_{6-77}=1,744 ; \mathrm{p}<0,05 ;$ número: $\mathrm{F}_{6-77}=1,313 ; \mathrm{p}$ $<0,05)$ (Figs 8 e 9).

As figuras 10 e 11 ressaltam as flutuações sazonais com diferenças significativas entre as CPUE $(\mathrm{kg})\left(\mathrm{F}_{3-80}=4,445 ; \mathrm{p}<\right.$ 0,05), (número) $\left(\mathrm{F}_{3-80}=8,860 ; \mathrm{p}<0,001\right)$ e as estações do ano. As diferenças ocorreram, principalmente, na primavera, onde foram registradas as maiores capturas sazonais.

\section{DISCUSSÃO}

A diferenciação sexual macroscópica foi possível a partir de exemplares com 11,0 cm de comprimento total, devido ao reduzido tamanho das gônadas em indivíduos menores, prin- 

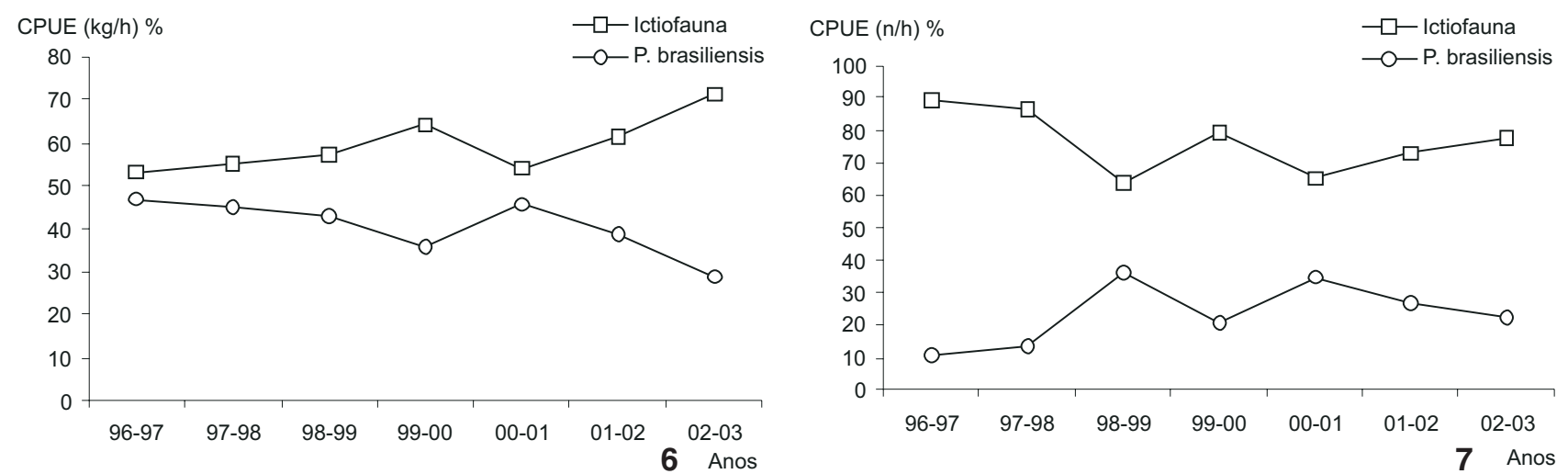

Figuras 6-7. Contribuição relativa em CPUE $\{\mathrm{kg} / \mathrm{h}$ (6), número de exemplares/h (7)\} de $P$. brasiliensis, na ictiofauna acompanhante da pesca do camarão sete-barbas, na Armação do Itapocoroy, no período de 1996 a 2003.
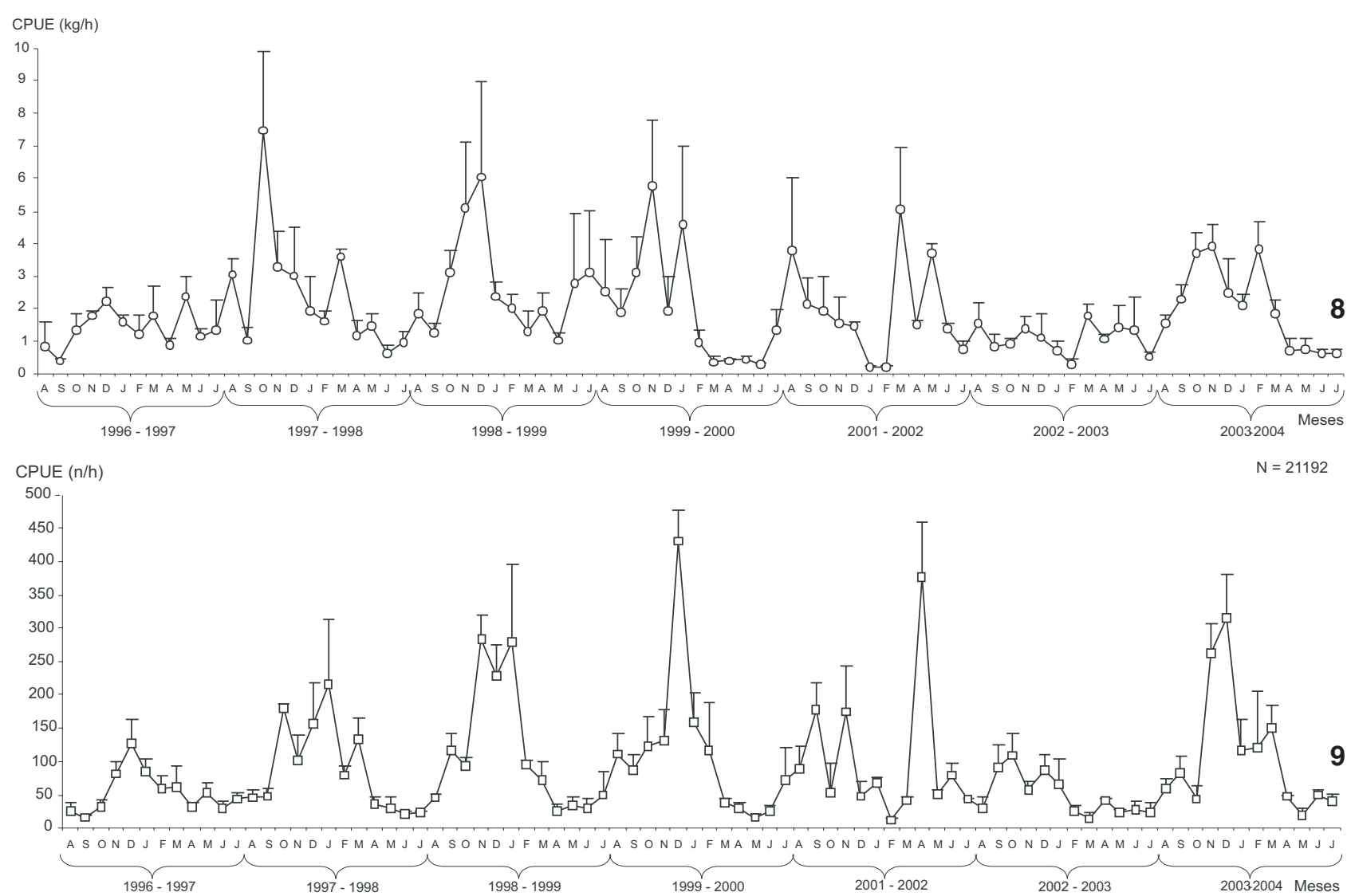

Figuras 8-9. Variação média mensal da CPUE $\{\mathrm{kg} / \mathrm{h}$ (8), número de exemplares/h (9)\} de $P$. brasiliensis, durante o período de 1996 a 2003. As barras verticais correspondem ao erro da média.

cipalmente fora da época de reprodução. Isso acarretou na ocorrência de exemplares de sexo indeterminado em grande parte das amostras, atingindo 34,4\%. CoеlHo et al. (1987), estudando um Sciaenidae de menor porte (Stellifer brasiliensis, Schultz,
1945), na pesca dirigida ao camarão sete-barbas no litoral de São Paulo, encontraram uma indefinição macroscópica das gônadas de peixes menores que $7,5 \mathrm{~cm}$ comprimento total, atingindo $36,2 \%$ dos exemplares capturados. 

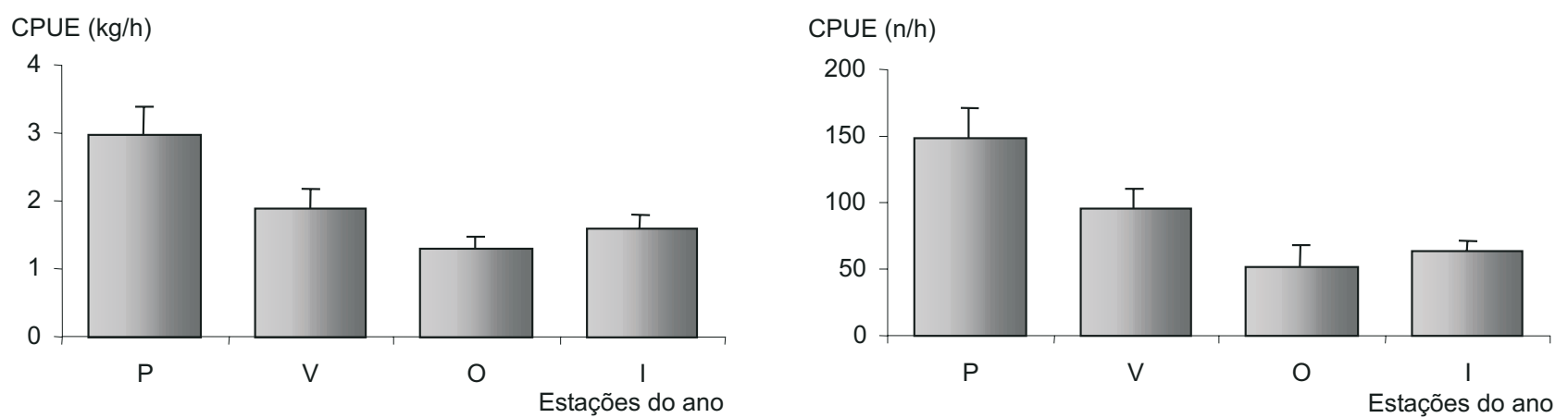

Figuras 10-11. Flutuação sazonal da CPUE média $\{\mathrm{kg} / \mathrm{h}(10)$, número de exemplares/h (11)\} de $P$. brasiliensis, nos sete anos de estudo. As barras verticais correspondem ao erro da média.

A proporção entre fêmeas e machos é uma informação importante para caracterização da estrutura de uma espécie ou população, além de fornecer subsídio para o estudo de outros aspectos bioecológico como a avaliação do potencial reprodutivo e estimativas do tamanho do estoque (Vazzoler 1996). P. brasiliensis apresentou uma dominância significativa de fêmeas em 11 meses da subamostragem. Comportamento similar foi observado por Cunningham \& Diniz FilHo (1995) na população do litoral de São Paulo. Para Coelho et al. (1987) o predomínio de fêmeas ao longo do ano, na pesca artesanal do camarão sete-barbas, acarreta em maior dano a população, pois a abundância dessas é um dos principais fatores que depende o potencial reprodutivo de uma população.

A determinação do tamanho de primeira maturação é fundamental para a administração racional dos estoques em exploração, fornecendo informações adequadas para estabelecer o tamanho mínimo de captura e conseqüente dimensionamento das malhas das redes (BRANCo et al. 2002). Dessa forma, a sobreposição desse tamanho com as curvas de distribuição de freqüência por comprimento permitem determinar o estrato da população em que a pesca vem atuando com maior intensidade (BrANCo et al. 1999). Assim, a pesca artesanal na Armação do Itapocoroy, parece estar atuando com maior intensidade sobre os exemplares adultos $(65,6 \%)$ de $P$. brasiliensis.

O tamanho de primeira maturação no litoral de São Paulo foi de 14,5 cm, sendo que a partir de 19,2 cm todos os exemplares coletados eram adultos (Cunninghan \& Diniz Filho 1995). Esses tamanhos, estão muito próximos dos obtidos nos presente estudo.

De acordo CoelHo et al. (1986) a pesca artesanal do camarão sete-barbas atua com maior intensidade sobre os juvenis, sendo que o comprimento médio dos Sciaenidae dominantes variou entre 8,5 e $11,3 \mathrm{~cm}$, esse tamanhos estão abaixo do registrado para $P$. brasiliensis nas subamostras da Armação do Itapocoroy.

A relação peso/comprimento, apresentou tendência exponencial, sendo o padrão de crescimento do tipo alométrico positivo. O mesmo padrão de crescimento foi encontrado para
Stellifer brasiliensis e S. rastrifer (Jordan, 1889) (GIANINNI \& PAIVAFilho 1995, Coelho et al. 1985, Gianinni \& Paiva-Filho 1990, Chaves \& Vendel 1997).

O estudo da alimentação natural em organismos aquáticos, evoluiu com o tempo, sendo desenvolvidas metodologias que atuam como ferramentas para auxiliar na avaliação e compreensão da disponibilidade dos itens presas consumidos (Lunardon-Branco \& Branco 2003). O método mais adequado a ser empregado para peixes, deve ser o que permita melhor compreender o espectro alimentar das espécies (Berg 1979). Existem poucas informações disponíveis sobre a bioecologia dos Sciaenidae no litoral brasileiro, especialmente enfocando os hábitos alimentares da espécie em estudo.

Não ocorreram diferenças significativas no volume relativo de alimento consumido por machos e fêmeas de $P$. bransiliensis. Comportamento semelhante foi observado na dieta natural do Sciaenidae Umbrina canosai Berg, 1895 na região do sul do Brasil (Haimovici et al. 1989).

Apesar das flutuações sazonais na dieta P. brasiliensis, os menores índices de repleção (outono-inverno) podem estar associados ao desenvolvimento das gônadas. Assim, o incremento na ingestão de alimento entre a primavera e o verão, provavelmente contribuem na preparação das gônadas na época de desova (Braga et al. 1985). Enquanto que para Paiva-Filho \& Rossi (1980), neste período a espécie necessita de alimento para recuperação das energias utilizadas na desova.

Paralonchurus brasiliensis apresentou um amplo espectro trófico, utilizando com maior freqüência os Polychaeta, Crustacea, Echinodermata e matéria orgânica; de acordo com esses componentes, pode ser considerada uma espécie de hábito bentônico. Para Haimovici et al. (1989) U. canosai utilizou 44 itens alimentares, sendo que Crustacea, Polychaeta, Echinodermata, Mollusca e Osteichthyes foram os recursos mais explorados. Enquanto que Menticirrhus littoralis (Holdrock, 1860) na região de Paranaguá-PR, utilizou 12 itens presas, com predomínio dos Crustacea, Bivalvia, Osteichthyes e Detritos, sendo considerada uma espécie de hábito bentônico (LuNARDon 1990). Esse padrão mostra que as espécies pertencentes à Famí- 
Tabela III. Freqüência sazonal de pontos (MP) dos itens alimentares de $P$. brasiliensis, e os índices de diversidade $\mathrm{H}^{\prime}$ e $\mathrm{H}_{\max }$

\begin{tabular}{|c|c|c|c|c|c|c|c|c|}
\hline \multirow{2}{*}{ Itens } & \multicolumn{2}{|c|}{ Primavera } & \multicolumn{2}{|c|}{ Verão } & \multicolumn{2}{|c|}{ Outono } & \multicolumn{2}{|c|}{ Inverno } \\
\hline & MP & $\%$ & MP & $\%$ & MP & $\%$ & MP & $\%$ \\
\hline Algae & & & & & & & 2 & 0,02 \\
\hline \multicolumn{9}{|l|}{ Cnidaria } \\
\hline Octocorallia & & & & & & & 25 & 0,22 \\
\hline Nematoda & & & 5 & 0,04 & 2 & 0,02 & & \\
\hline \multicolumn{9}{|l|}{ Mollusca } \\
\hline Pitar fulminatus & & & & & & & 17 & 0,15 \\
\hline \multicolumn{9}{|l|}{ Polychaeta } \\
\hline Flabelligeridae & 4340 & 44,57 & 6190 & 53,28 & 3520 & 41,79 & 3992 & 35,31 \\
\hline Nereidae & 100 & 1,03 & 465 & 4,00 & 257 & 3,05 & 40 & 0,35 \\
\hline Eunicidae & & & & & & & 32 & 0,28 \\
\hline Ophelidae & & & & & & & 125 & 1,11 \\
\hline Lumbrineridae & & & & & & & 29 & 0,26 \\
\hline Polychaeta & 975 & 10,01 & 637 & 5,48 & 235 & 2,79 & 1454 & 12,86 \\
\hline \multicolumn{9}{|l|}{ Crustacea } \\
\hline Copepoda & & & 10 & 0,09 & & & & \\
\hline Acetes americanus & 810 & 8,32 & & & 1525 & 18,11 & 1325 & 11,72 \\
\hline Pleoticus müllieri & 100 & 1,03 & 761 & 6,55 & & & 150 & 1,33 \\
\hline Dendrobranchiata & 1023 & 10,51 & 880 & 7,57 & 522 & 6,20 & 355 & 3,14 \\
\hline Brachyura & 354 & 3,64 & 344 & 2,96 & & & 52 & 0,46 \\
\hline Pinnotheridae & 450 & 4,62 & 42 & 0,36 & 485 & 5,76 & 2546 & 22,52 \\
\hline Callinectes & & & & & & & 30 & 0,27 \\
\hline Misidacea & 57 & 0,59 & & & & & 54 & 0,48 \\
\hline Cumacea & 16 & 0,16 & 302 & 2,60 & 14 & 0,17 & 2 & 0,02 \\
\hline Isopoda & & & 50 & 0,43 & & & 42 & 0,37 \\
\hline Gammaridae & 365 & 3,75 & 16 & 0,14 & 381 & 4,52 & 260 & 2,30 \\
\hline Stomatopoda & 50 & 0,51 & & & & & & \\
\hline Ovos de Crustacea & & & & & & & 2 & 0,02 \\
\hline \multicolumn{9}{|l|}{ Echinodermata } \\
\hline Ophiuroidea & 276 & 2,83 & 521 & 4,48 & 296 & 3,51 & 352 & 3,11 \\
\hline Osteichthyes & 235 & 2,41 & 275 & 2,37 & & & 75 & 0,66 \\
\hline Matéria Orgânica & 512 & 5,26 & 1108 & 9,54 & 1186 & 14,08 & 344 & 3,04 \\
\hline Areia & 75 & 0,77 & 12 & 0,10 & & & & \\
\hline Média dos pontos & 512,52 & 100,00 & 726,12 & 100,00 & 765,72 & 100,00 & 434,81 & 100,00 \\
\hline N. itens utilizados & 16 & & 16 & & 11 & & 23 & \\
\hline $\mathrm{H}_{(\text {nats) }}^{\prime}$ & 1,9806 & & 1,7178 & & 1,7634 & & 1,9356 & \\
\hline $\mathrm{H}_{\max (\text { nats })}$ & 2,8332 & & 2,7725 & & 2,3978 & & 3,1780 & \\
\hline$E=J^{\prime}(\%)$ & 69,90 & & 61,95 & & 73,54 & & 60,90 & \\
\hline
\end{tabular}

lia Sciaenidae apresentam um amplo espectro trófico, onde os Polychaeta e Crustacea desempenham um papel fundamental na dieta dos peixes. Amaral \& Migotto (1980) confirmam a importância dos poliquetas na dieta de P. brasiliensis capturados na região de Ubatuba (São Paulo).
A captura por unidade de esforço (CPUE) em biomassa ou número de exemplares é o índice de abundância mais adequado nos monitoramentos pesqueiros. Mudanças nesse índice refletem alterações temporais na abundância de peixes, como as observadas neste estudo. Apesar das flutuações sazonais nas 
taxas de captura de $P$. brasiliensis, estudos como o presente, analisando uma série temporal de dados são raros e de grande interesse, principalmente, tratando-se de um recurso com potencial econômico e relevância sócio-cultural no litoral brasileiro.

\section{AGRADECIMENTOS}

À Universidade do Vale do Itajaí - UNIVALI, através do Centro de Ciências Tecnológicas, da Terra e do Mar - CTTMar, pelas facilidades colocadas à disposição durante o andamento deste trabalho. Ao Oc. Flávio Souto Xavier, nossos sinceros agradecimentos pela coleta dos dados de alimentação. Aos exorientandos e estagiários: MSc. Hélio Augusto Alves Fracasso; Oceanógrafos: Gislei Cibele Bail, Laura Ribas de Almeida, Heder Cassiano Moritz Junior, Rodrigo Schveitzer, Willian Gimarães Vale, Jan Raphael Reuter Braun, Bruno Ribeiro Campos, entre outros, pelo valioso auxílio nos trabalhos de campo.

\section{REFERÊNCIAS BIBLIOGRÁFICAS}

Amaral, A.C.Z. \& A.E. Migoto. 1980. Importância dos anelídeos poliquetas na alimentação da macrofauna demersal e epibentônica da região de Ubatuba. Boletim do Instituto Oceanográfico, São Paulo, 29 (2): 31-35.

BERG, J. 1979. Discussion of methods of investigating the food of fiches, with a reference to a preliminary study of the prey of Gobiusculus flavencens (Gobiidae). Marine Biology, Berlin, 50: 263-273.

Braga, F.M.S.; M.A.S. Braga \& S. Goitein. 1985. Fator de condição e alimentação de Paralonchurus brasiliensis (Osteichthyes,

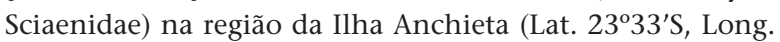
$45^{\circ} 05^{\prime} \mathrm{W}$ ) Ubatuba, Estado de São Paulo. Naturalia, São Paulo, 10: 1-11.

Branco, J.O; M.J. Lunardon-Branco; F.X. Souto \& C.R. Guerra. 1999. Estrutura populacional do camarão sete-barbas Xiphopenaeus kroyeri (Heller, 1862), na foz do rio Itajaí-Açú, Itajaí, SC, Brasil. Brazilian Archives of Biology and Technology, Curitiba, 42 (1): 115-126.

Branco, J.O.; M.J. Lunardon-Branco \& F.X. Souto. 2002. Estrutura populacional de Portunus spinimanus Lateille (Crustacea, Portunidae) na Armação do Itapocoroy, Penha, Santa Catarina, Brasil. Revista Brasileira de Zoologia, Curitiba, 19 (3): 731-738.

Chaves, P.T.C. \& A.L. Vendel. 1997. Reprodução de Stellifer rastrifer (Jordan) (Teleostei, Sciaenidae) na baía de Guaratuba, Paraná, Brasil. Revista Brasileira de Zoologia, Curitiba, 14 (1): 81-89.

Coelho, J.A.P.; R. Graça-Lopes; E.S. Rodrigues \& A. Puzzi. 1985. Relação peso-comprimento e tamanho de início de primeira maturação gonadal para o Sciaenidae Stellifer rastrifer (Jordan, 1889), no litoral do Estado de São Paulo. Boletim do Instituto de Pesca, São Paulo, 12 (2): 99-107.

Coelho, J.A.P.; A. Puzzi; R. Graça-Lopes; E.S. Rodrigues \& Jr.O.
Preto. 1986. Análise da rejeição de peixes na pesca artesanal dirigida ao camarão sete-barbas (Xiphopenaeus kroyeri) no litoral do Estado de São Paulo. Boletim do Instituto de Pesca, São Paulo, 13 (2): 51-61.

Coelho, J.A.P.; R. Graça-Lopes; E.S. Rodrigues \& A. Puzzi. 1987. Aspectos biológicos e pesqueiros do Sciaenidae Stellifer brasiliensis (Schultz, 1945), presente na pesca artesanal dirigida ao camarão sete-barbas (São Paulo, Brasil). Boletim do Instituto de Pesca, São Paulo, 14: 1-10.

Cunningham, P.T.M. \& A.M. Diniz Filho. 1995. Aspectos da biologia de Paralonchurus brasiliensis, Sciaenidae - no litoral norte de São Paulo, Brasil. Boletim do Instituto Oceanográfico, São Paulo, 11: 203-210.

Gianinni, R. \& A.M. Paiva-Filho. 1990. Aspectos bioecológicos de Stellifer rastrifer (Perciformes: Sciaenidae) na Baía de Santos, SP. Boletim do Instituto de Pesca, São Paulo, 38 (1): 57-67.

Gianinni, R. \& A.M. Paiva-Filho. 1995. Distribuição temporal, espacial e bioecologia do cangoá, Stellifer brasiliensis (Teleostei: Sciaenidae), na Baía de Santos, São Paulo, Brasil. Arquivos de Ciências do Mar, Fortaleza, 29 (1-2): 5-15.

Haimovici, M.; R.L. Teixeira \& M.C. Arruda. 1989. Alimentação da Castanha Umbrina canosai (Pisces: Scieanidae) no Sul do Brasil. Revista Brasileira de Biologia, Rio de Janeiro, 49 (2): 511-522.

Hynes, H.B.N. 1950. The food of fresh-water stiklebacks (Gastersteus aculeatus and Pygosteus pungitius) with a review of method used in studies of the food fishes. Journal Animal Ecology, London, 19 (1): 36-51.

HysLop, E.J. 1980. Stomach contents analysis - a review of methods and their application. Journal of Fish Biology, London, 17: 411-429.

KaWAKAMI, E. \& G. VAzzoler. 1980. Método gráfico e estimativa de índice alimentar aplicado no estudo de alimentação de peixes. Boletim do Instituto Oceanográfico, São Paulo, 29 (2): 205-207.

LudWIG, J.A. \& J.F. REYNOLDs. 1988. Statistical ecology: a primer on methods and computing. New York, John Wiley \& Sons, 338p.

Lunardon, M.J. 1990. Hábitos alimentares de Menticirrhus littoralis (Holdrock, 1860) (Perciformes - Sciaenidae) na Baía de Paranaguá e adjacências, Paraná, Brasil. Arquivos de Biologia e Tecnologia, Curitiba, 33 (3): 717-725.

Lunardon-Branco, M.J. \& J.O. Branco. 2003. Alimentação natural de Etropus crossotus Jordan \& Gilbert (Teleostei, Pleuronectiformes: Paralichthyidae), na Armação do Itapocoroy, Penha, SC, Brasil. Revista Brasileira de Zoologia, Curitiba, 20 (4): 631-635.

Menezes, N.A. \& J.L. Figueiredo. 1980. Manual de peixes marinhos do sudeste do Brasil: IV Teleostei (3). São Paulo, Museu de Zoologia, Universidade de São Paulo, 96p.

Paiva-Filho, A.P. \& L. Rossi. 1980. Estudo sobre a fecundidade e a desova de Paralonchurus brasiliensis (Steindachner, 1875), 
população de SP (Osteichthyes, Sciaenidae). Revista Brasileira de Biologia, Rio de Janeiro, 40 (2): 241-247.

Paiva-Filho, A.M. \& J.M.M. Schmiegelow. 1986. Estudo sobre a ictiofauna acompanhante da pesca do camarão sete-barbas (Xiphopenaeus kroyeri) nas proximidades da Baía de Santos, SP. 1. Aspectos quantitativos. Boletim do Instituto Oceanográfico, São Paulo, 34: 78-85.

Paiva-Filho, A.M. \& M.L. Zani-Teixeira. 1980. Estudo da sobreposição de Paralonchurus brasiliensis (Steindachner, 1875) na costa sudeste-sul do Brasil entre as latitudes $22^{\circ} 10^{\prime} \mathrm{S}$ e $29^{\circ} 21^{\prime} \mathrm{S}$ (Osteichthyes, Sciaenidae). Revista Brasileira de
Biologia, Rio de Janeiro, 40 (1):143-148.

SANTOS, E.P. 1978. Dinâmica de populações aplicada à pesca e piscicultura. São Paulo, HUCITEC/EDUSP, 129p.

SOKAL, R.R. \& F.J. RoHLF. 1969. Biometry, the principles and practies of statistics in biological research. San Francisco, W.H. Freeman, 776p.

VAzzoler, A.E.A.M. 1981. Manual de métodos para estudos biológicos de populações de peixes: reprodução e crescimento. Brasília, CNPq, Programa Nacional de Zoologia, 106p.

VAZzolER, A.E.A.M. 1996. Biologia da reprodução de peixes teleósteos: teoria e prática. Maringá, EDUEM, 169p.

Recebido em 11.IV.2005; aceito em 07.XI.2005. 